\title{
Hagiographie, idéologies cléricales et pratiques
} sociales au Moyen Âge

Conférences de l'année 2011-2012

Patrick Henriet

\section{(2) OpenEdition}

\section{Journals}

Édition électronique

URL : https://journals.openedition.org/ashp/1499

DOI : 10.4000/ashp.1499

ISSN : 1969-6310

\section{Éditeur}

Publications de l'École Pratique des Hautes Études

Édition imprimée

Date de publication : 1 septembre 2013

Pagination : 155-157

ISSN : 0766-0677

Référence électronique

Patrick Henriet, « Hagiographie, idéologies cléricales et pratiques sociales

au Moyen Âge », Annuaire de l'École pratique des hautes études (EPHE), Section des sciences historiques et philologiques [En ligne], 144 | 2013, mis en ligne le 24 octobre 2014, consulté le 05 novembre 2021. URL : http://journals.openedition.org/ashp/1499 ; DOI : https://doi.org/10.4000/ashp.1499 


\title{
HAGIOGR APHIE, IDÉOLOGIES CLÉRICALES ET PRATIQUES SOCIALES AU MOYEN ÂGE
}

\author{
Directeur d'études : M. Patrick Henriet
}

Programme de l'année 2011-2012: I. Le Liber miraculorum sancti Isidori de Lucas de Tuy (années 1220-1230), chapitres 17-30. - II. Vie et visions d'Alpais de Cudot († 1211), les visions. - III. Conférences et exposés.

Les deux dossiers ouverts en 2010-2011 (voir leur présentation dans l'Annuaire 2010-2011) ont été à nouveau étudiés cette année, avec une priorité pour le Liber miraculorum de Lucas de Tuy. La première partie de chaque séance a été consacrée à l'« actualité de la recherche » en hagiologie, avec le compte rendu de divers ouvrages et articles.

I. Le Liber miraculorum sancti Isidori de Lucas de Tuy (années 1220-1230), chapitres 17-30

Il a été possible de lire cette année, de façon extrêmement détaillée, quatorze chapitres du recueil. La notion même de « chapitre » a été remise en cause, car il s'agit de savoir si l'actuelle capitulation remonte bien au XIII ${ }^{\mathrm{e}}$ siècle et à Lucas. De forts doutes sont exprimés, mais un examen attentif et systématique de cette question est prévu pour l'année 2012-2013, qui devrait aussi voir la fin de l'étude du LMSI (encore inédit dans sa version latine, rappelons-le). Les miracles étudiés cette année permettent à Lucas de s'émanciper pleinement de ses devanciers, et en particulier de l'Historia translationis (BHL 4491). Tous ces textes (de même d'ailleurs que, déjà, le miracle 16) rapportent ben effet des événements qui ne sont connus que grâce à Lucas. Ils constituent deux gros blocs thématiques d'un intérêt souvent exceptionnel.

Un premier ensemble traite des miracles accomplis par Isidore à Séville après la translation de son corps à León en 1063 (chapitres 17-23). Il y a là matière à de beaux morceaux de propagande anti-musulmane en même temps qu'à un discours sur la virtus du saint, qui demeure présente sur le lieu de son ancien sépulcre alors même que son corps en est absent. Mais on retiendra aussi que ces textes donnent un grand nombre de précisions extrêmement intéressantes sur les rapports entre chrétiens et musulmans ainsi que sur la possible persistance d'une petite communauté chrétienne à Séville à la fin du XII ${ }^{\mathrm{e}}$ siècle (ce qui contredit en partie l'état actuel de la recherche). Lucas mentionne sa source principale, à savoir le grand aristocrate Pedro Fernández de Castro, qui avait résidé à Séville après avoir dû s'exiler chez l'adversaire musulman et qui était un dévot d'Isidore. Cet ensemble de textes possède indéniablement une tonalité très spécifiquement hispanique. Isidore va jusqu'à guérir des musulmans qui affirment croire en sa virtus, leur conversion n'ayant lieu qu' a posteriori. On relèvera 
enfin, parmi d'autres, ce passage où Lucas se met lui-même en scène alors qu'il tente d'acheter une lampe qui brûlait miraculeusement dans le sépulcre d'Isidore et qui avait été rapportée à León.

Un deuxième ensemble de textes (chapitres 25-30) décrit le pillage de Saint-Isidore par les troupes d'Alphonse le Batailleur, roi d'Aragon, dans la deuxième décennie $\mathrm{du} \mathrm{XII}^{\mathrm{e}}$ siècle, lors des luttes qui l'opposèrent à son épouse Urraca. La lecture attentive de ces chapitres a permis de se livrer à quelques réflexions assez poussée sur la matérialité du reliquaire d'Isidore et sur la façon dont il était conservé dans les décennies qui suivirent la translation. On a ainsi pu émettre, textes à l'appui, l'hypothèse que cette œuvre extraordinaire, aujourd'hui conservée à Madrid, avait originellement été placée dans une arca qui contenait aussi les reliques de saint Vincent d'Avila. On connaissait certes déjà l'existence d'une telle arca grâce à une description manuscrite $\mathrm{du} \mathrm{XIX}^{\mathrm{e}}$ siècle, mais le texte de Lucas permet de comprendre, ou en tout cas de supposer, que l'arca ( $\mathrm{XII}^{\mathrm{e}}$ siècle très certainement) détruite à l'époque de l'invasion napoléonienne avait été précédée d'une autre arca dès la fin $\mathrm{du} \mathrm{XI}^{\mathrm{e}}$ siècle, laquelle disparut lors des exactions d'Alphonse le Batailleur. Si cette reconstitution est exacte, on doit donc imaginer dès la fin du XI ${ }^{\mathrm{e}}$ siècle un dispositif complexe associant autel, arca, reliquaires et sans doute croix. Saint-Isidore aurait ainsi sa place auprès d'ensembles du même type beaucoup mieux connus et documentés, comme ceux d'Oviedo et de Silos.

Enfin, une séance a été consacrée à la présentation d'un autre texte inédit relatif à saint Isidore : il s'agit d'un éloge très largement inspiré de la Vita sancti Isidori (BHL 4486), présent dans un manuscrit tolédan du XIII ${ }^{\mathrm{e}}$ siècle. Cette œuvre est attribuable (grâce à une mention de Lucas de Tuy dans le Liber miraculorum) à Pedro Muñiz $(† 1224)$, diacre puis évêque de León avant de devenir archevêque de Compostelle. Le texte peut être daté assez précisément entre 1196 et 1206 . L'hypothèse a même été avancée que Pedro Muñiz pourrait être aussi l'auteur de la Vita sancti Isidori, que l'on peut maintenant (et en partie grâce à cette découverte) dater des années 1186-1206.

\section{Vie et visions d'Alpais de Cudot $(† 1211)$, les visions}

La Vita d'Alpais de Cudot (BHL 306) avait déjà été abordée l'an dernier. Cette année, après avoir vu la Vita proprement dite, nous nous sommes concentrés sur l'activité visionnaire de la sainte. L'auteur, un moine cistercien anonyme de l'abbaye des Écharlis qui écrit à la fin du XII ${ }^{\mathrm{e}}$ siècle, explique longuement la façon dont Alpais recevait ses révélations. Il en donne la teneur et décrit de façon saisissante les relations entre la recluse et son entourage. Deux particularités du recueil ont été soulignées : d'une part la spécialisation d'Alpais dans ce qu'on appelle parfois les « visions cosmiques » (raison pour laquelle cette sainte est aujourd'hui patronne des cosmonautes). On n'en dénombre pas moins de quatre dans le recueil, chacune d'entre elles poursuivant une visée pédagogique et ecclésiologique très marquée. Alpais s'élève dans les cieux et voit l'ensemble de la terre, laquelle est marquée par des églises, des tombes, etc.

D'autre part, on a pu mettre en valeur deux passages (l'un du cistercien anonyme auteur des quatre livres, l'autre étant écrit par un chanoine continuateur $=$ BHL 307) qui donnent une étonnante illustration littéraire et quasi visuelle du thème relativement classique de «l'abbé nourricier»: des hommes (un prêtre, un abbé) apparaissent en 
vision à Alpais et donnent le sein aux populations dont ils ont la charge. Il n'est pas certain que l'on trouve des scènes de ce type dans l'hagiographie antérieure à la fin du $\mathrm{XII}^{\mathrm{e}}$ siècle et on voit là, dans une œuvre peu connue mais qui a tout de même eu une certaine diffusion, la mise en forme d'une spiritualité affective qui semble assez propre aux cisterciens. Ce dernier point a été commenté, textes à l'appui, lors d'une excursion effectuée à Cudot (Yonne) pour marquer la fin des séminaires en juin. Cette journée a permis de réunir plusieurs auditeurs et a permis de visiter l'église de Cudot (où l'on a pris connaissance d'un récent vol de reliques accompli pour des raisons non commerciales), mais aussi les quelques restes du monastère des Écharlis et les ruines de l'abbaye, cistercienne, elle aussi de Fontainejean (Loiret), plusieurs fois mentionnée par notre hagiographe anonyme.

\section{Conférences et exposés}

Plusieurs conférences ou exposés ont été données ou présentés dans le cadre du séminaire. Raquel Alonso (université d'Oviedo), spécialiste reconnue de l'art roman hispanique et de Pélage, évêque d'Oviedo au début du XII ${ }^{\mathrm{e}}$ siècle, a parlé de " L'Arca Santa de la cathédrale d'Oviedo : réélaborer, resituer, manipuler ». Florence Chave, auteur d'un récent beau livre sur L'exorcisme des possédés dans l'Église d'Occident ( $X^{e}-X I V^{e}$ siècle), Turnhout, 2011, a présenté son travail en commentant divers textes. Marguerite Piot (M2) a donné un aperçu de ses recherches sur « Le miracle chez Pierre Damien ». Alexandre Romain-Desfossés (M2) a présenté son travail sur «Ascétisme et combat spirituel dans la Vie de Bernard de Tiron (BHL 1251) ». Pauline Bouchaud (M2), enfin, a fait de même autour de « Étienne Maleu († 1322), hagiographe ». 